This PDF is a selection from a published volume from the National Bureau of Economic Research

Volume Title: Economic Dimensions of Personalized and Precision Medicine

Volume Authors/Editors: Ernst R. Berndt, Dana P. Goldman, and John W. Rowe, editor

Volume Publisher: University of Chicago Press

Volume ISBNs: 978-0-226-61106-8 (cloth); 978-0-226-61123-5 (electronic)

Volume URL: http://www.nber.org/books/bern-13

Conference Date: September 13-14, 2017

Publication Date: April 2019

Chapter Title: The Economic Value and Pricing of Personalized Medicine Chapter Author(s): Tomas J. Philipson

Chapter URL: http://www.nber.org/chapters/c13997

Chapter pages in book: (p. $9-19)$ 


\title{
The Economic Value and Pricing of Personalized Medicine
}

\author{
Tomas J. Philipson
}

\subsection{Introduction}

Personalized medicine (PM) entails better tailoring therapies to patients through use of information about the patient that better predicts treatment response before undergoing treatment. Personalized medicine is rapidly emerging; currently, about 130 Food and Drug Administration (FDA)approved drugs have pharmacogenomic information in their labeling (FDA 2017). Personalized medicine will likely continue to grow in the future; Tufts (2015) has estimated that the PM share grew from 5 percent of the FDA pipeline in 2005 to 25 percent in 2015.

Since assigning the correct treatment for a given diagnosis has been a goal of medicine since its inception, the development of PM is just a more recent version or continuation of this long-run trend. Providers have always sought to use available information, for example, gender, age, race, symptoms, or laboratory tests to determine whether a given treatment is likely to be useful for a given patient. What is new about recent PM innovations in the ongoing quest to better match appropriate treatments to patients is that they involve treatment-specific diagnostics, called companion diagnostics, used to test the patient into or out of a given therapy for a given diagnosis.

Tomas J. Philipson was the Daniel Levin Professor of Public Policy Studies at the University of Chicago Harris School of Public Policy and a director of the Health Economics Program of the Becker Friedman Institute at the University when this chapter was written. He is currently serving as a member of the Council of Economic Advisers at the White House.

I am thankful to seminar participants at the University of Chicago and NBER for comments, and especially Ernst Berndt and Thomas McGuire for comments that improved the chapter. Financial support from the NBER and the Becker Friedman Institute at the University of Chicago is gratefully acknowledged. For acknowledgments, sources of research support, and disclosure of the author's material financial relationships, if any, please see http://www.nber .org/chapters/c13997.ack. 
Companion diagnostics based on genetic tests have diffused rapidly as the cost of sequencing has gone from about $\$ 300$ million to $\$ 1,000$ in the last fifteen years. Even though diagnostics have become treatment specific, there is not so much conceptually different in PM compared to the historical conduct of testing for a diagnosis and prescribing medicines conditional on the diagnosis. Testing cholesterol levels to determine which patients are appropriate for statins is in principle the same type of medical practice as using gene tests to determine which breast cancer patients are appropriate for a given cancer drug. Both involve only wanting to assign a treatment to a patient for which it will be of value.

This chapter discusses the economic value of such PMs as well as the optimal pricing of the combination products involved. We build on the work of Egan and Philipson (2015) and Sood, Philipson, and Huckfeldt (2013), who argue that there is a close link between rational nonadherence in health care and the value of personalized medicine. This stems from interpreting adherence as a simple learning problem for a patient learning about the individual value of a therapy. Although providers recommending treatments are likely more informed about the population-wide effects of these treatments, patients experiencing a treatment are more informed about the individualspecific value of a treatment. This individual-specific value of a treatment incorporates how the patient trades off patient-specific treatment effectiveness, side effects, and costs of care and other factors. In that process, a patient's prior beliefs about a treatment coupled with the patient's experience with the treatment drive initiation and subsequent adherence. The patient behavior mimics the common-sense approach of patients using a treatment, assessing its value on an ongoing basis, and discontinuing treatment if it is not valuable. Nonadherence is thus inherently a dynamic demand behavior that requires an explanation of why people initiate but then discontinue therapy, and learning about treatment value provides one natural explanation.

As the patient is learning about treatment value through experiencing the treatment, there may be type I and II errors in adherence; a patient may drop treatment when they should not and may stay on treatment when they should not. This leads to inefficient care; the first error leads to underconsumption of the treatment and the second to overconsumption. We argue that the emergence of PM is best interpreted as a valuable technological change aimed at reducing such inefficiencies. Personalized medicine reduces consumption for nonresponders and raises it for responders.

Testing for treatment value before undertaking therapy involves changing the therapy from an experience good, for which consumption experience is required to determine its quality, to a search good, for which it is not. Companion diagnostics in PM essentially convert experience goods to search goods by speeding up the learning process and cutting both type I and II errors in adherence. The emergence of valuable technological change in terms of innovations in personalized medicine is therefore explained by 
information and learning as being central to adherence. If learning were not important for adherence, then there would be no value from companion diagnostics. In particular, the value of PM is greater when learning through experience is costly relative to learning through a diagnostic. This offers a key explanation as to why the emergence of PM has taken place disproportionately in cancer care, where type I and II errors in adherence may be very large; nonresponders being on the wrong treatment while a tumor is growing may have fatal outcomes. It also helps explain the timing of emerging PM in cancer care, where many new treatments raise the opportunity cost of being on the wrong treatment.

The outline of this chapter is as follows. Section 1.2 briefly reviews the relationship between adherence and PM, as emphasized by Egan and Philipson (2015), and considers a simplified version of that analysis to derive the value of PM and how its value is allocated across the patient population testing into or out of a given treatment. Section 1.3 considers the unusual aspects of the optimal pricing of PM, and these resulting incentives for innovation include how mergers between drugs $(\mathrm{Rx})$ and diagnostics (Dx) producers may enhance optimal pricing and innovation. Last, section 1.4 concludes.

\subsection{Learning about Individual Treatment Value through Experience}

We first summarize the main arguments of Egan and Philipson (2015) on learning and rational adherence, with the latter analysis relying on a simplified version of that framework. The analysis argues that adherence to treatments reflects revealed preference under a learning process about the individual treatment value of the patient, and that PM speeds up that learning to take place before consumption rather than through consumption.

Egan and Philipson interpret nonadherence as a simple optimal stopping problem for a patient learning about his individual value of a therapy analogous to optimal adherence in clinical trials analyzed by Philipson and Desimone (1997). Although providers recommending treatments are likely more informed about the population-wide effects of treatments, patients experiencing a treatment are more informed about the individual-specific value of treatment. This individual-specific value of treatment incorporates how the patient trades off patient-specific treatment effectiveness, side effects, and costs of care.

A patient's prior beliefs about a treatment, how optimistic he is it will work, coupled with the patient's experience with the treatment, drive initiation and subsequent adherence. With each observation drawn on the treatment, say through each subsequent dose, the patient places more weight on his treatment experience and less on his prior. In addition, his posterior variance decreases over time, making him more confident about the patientspecific treatment value. The patient behavior mimics the common-sense 
approach of starting to use a treatment, assessing its value while on it, and discontinuing treatment if he learns it is not valuable. Nonadherence is thus inherently a dynamic demand behavior that requires an explanation of why people initiate but then discontinue therapy. Learning about treatment value provides one natural explanation.

Egan and Philipson (2015) derive the adherence behavior conditional on a patient type $(q)$ as being characterized by the survival function $S(t \mid q)$, which reflects the proportion of type $q$ individuals still remaining on or adhering to the treatment at time $t$. The overall survival function of adherence thus results from aggregating over all types

$$
S(t)=\int S(t \mid q) d F(q) \text {. }
$$

Figure 1.1 depicts such empirical survival functions often reported in the medical literature across several alternative treatment classes. It displays the general pattern of nonadherence occurring early but then stabilizing, which reflects patients learning about treatment value initially and then stabiliz-

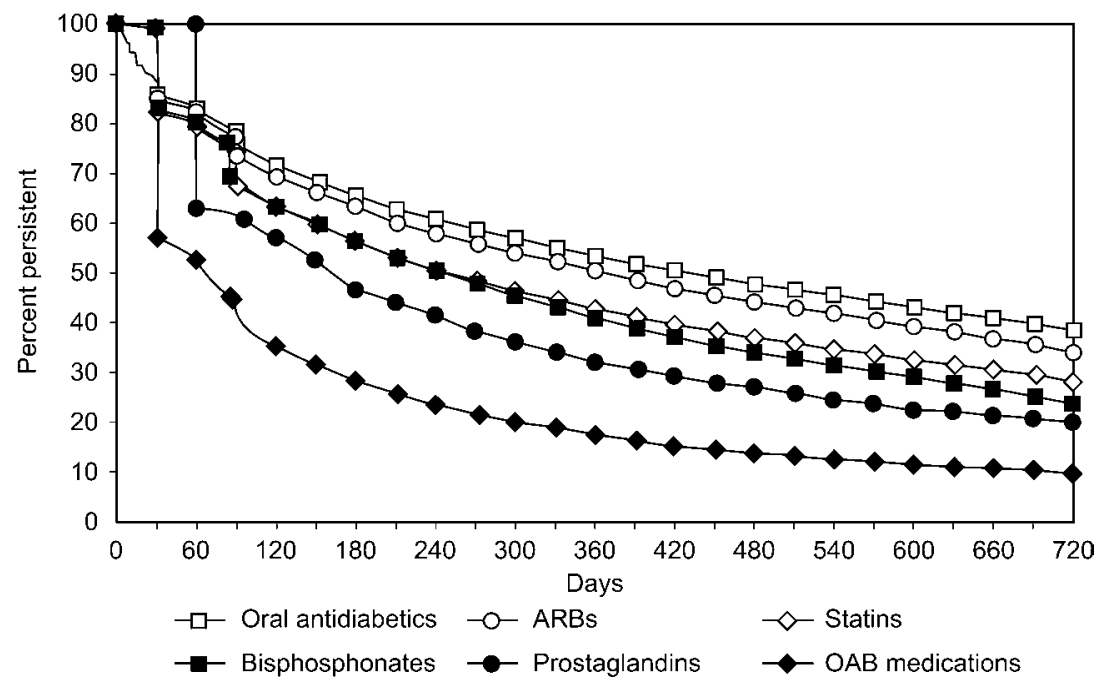

Fig. 1.1 Adherence survivals across treatment classes

Source: This figure is taken directly from Yeaw et al. (2009, figure 2).

Notes: The figure illustrates observed adherence patterns for prostaglandin analogs, statins, bisphosphonates, oral antidiabetics, angiotensin II receptor blockers (ARBs), and overactive bladder $(\mathrm{OAB})$ medications. Details for each drug cohort are as follows. Prostaglandin analogs, such as latanoprost (Xalatan), are used to manage glaucoma. Statins, such as atorvastatin (Lipitor), are used to lower cholesterol levels. Bisphosphonates, such as alendronate sodium (Fosamax), are used to treat osteoporosis. Oral antidiabetics, such as metformin (Glucophage), are used to control blood sugar levels for patients with type 2 diabetes. Angiotensin II receptor blockers, such as losartan potassium (Hyzaar) and hydrochlorothiazide, are used to treat hypertension. The OAB medications, such as tolterodine tartrate (Detrol LA), are used to treat overactive bladders. 
ing after such learning has taken place, with the true value of the treatment being established through experience replacing priors in driving adherence.

As the patient learns about treatment value through experiencing the treatment, type I and II errors in adherence may emerge; a patient may drop treatment when he should not and may stay on a treatment when he should not. Figure 1.2 illustrates the general pattern of both types of errors in adherence. The survival curve $S^{V}(t)$ reflects the proportion of those types for whom the treatment turns out, indeed, to be valuable. The shaded area above this survival, for those who actually value the treatment, thus reflects the share engaged in type I errors, lack of adherence or consumption when there should be consumption. Inefficient underadherence occurs because some of the patients experience poor initial performance on the treatment and discontinue even though the treatment is in fact valuable.

The survival curve $S^{N}(\cdot)$ reflects the proportion of those who turn out not to value the treatment but still adhere. Such inefficient overadherence is reflected by type II errors - consumption when there should not be - and is given by the area below this survival curve. However, sooner or later all of them will learn that the treatment is not valuable, so no one adheres; $\lim _{t \rightarrow \infty} S^{N}(t)=0$. The overall observed survival curve is the unobservable mixture of the unobservable survivals of the two groups. The dotted line in figure 1.2 reflects the ex post optimal adherence level without type I and II errors, which is simply the fraction of the population that responds to treatment, assumed to be half in the figure.

Personalized medicine represents a technological change or innovation

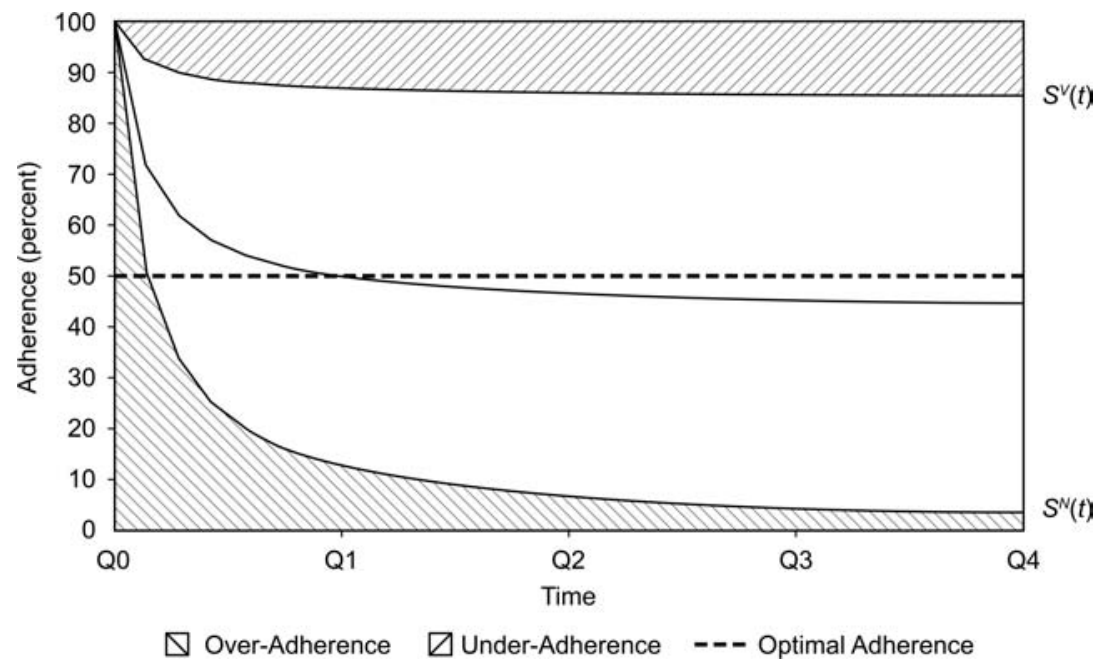

Fig. 1.2 Adherence behavior by those who turn out to value or not value the treatment 
that reduces both shaded areas in the figure by raising adherence or consumption for those who test into therapy, while lowering the adherence by those who test out of therapy. Personalized medicine, by use of companion diagnostics, speeds up learning about treatment value compared to learning through trial and error or experience with the therapy. It thus amounts to trying to eliminate the under- and overconsumption associated with the shaded regions in figure 1.2.

To illustrate the implications of this analysis for the value and pricing of PM, we consider a simplified version of the learning environment above. Let $P$ and $p$ denote the respective prices of the companion diagnostic and the treatment. We consider two groups that value or do not value the treatment and denote the fractions of each by $(R, 1-R)$, respectively. We refer to the two groups as responders and nonresponders, although response may not fully be determined by clinical response differences but rather by value differences taking into account more treatment dimensions than just clinical effectiveness. The responders are defined to value the treatment by a positive amount $V$. For the nonresponders, this value is a negative amount or cost $C$, which includes, among other things, forgone health from not being on the correct alternative treatment. This may be a large cost to nonresponders, particularly in cancer, explaining why PM has occurred there with an emerging large set of treatments and high costs of being on the wrong therapy.

The expected utility from undergoing treatment without the help of a companion Dx is then

$$
E\left[U_{R_{x}}\right]=R \cdot V+(1-R) \cdot C-p .
$$

There are two cases to consider, corresponding to whether this expected utility of being on treatment is larger or smaller than for the alternative care, the latter assumed zero without loss of generality.

Consider first when the expected utility of the treatment is positive so that the treatment is undertaken, rather than alternative care, in absence of personalized medicine. Now consider when there is a companion diagnostic that allows the patient to determine at the start before undergoing treatment whether he will respond to the treatment. The patient then only undertakes and pays for the underlying treatment if he tests into it, and opts out when he tests out. This yields expected utility with the diagnostic

$$
E\left[U_{D_{x}}\right]=R \cdot(V-p)-P .
$$

The value of testing is the consumer surplus when testing in, less the cost of the test. Figure 1.3 illustrates the value of PM as a function of the response rate. The patient will undertake PM, $E\left[U_{D_{x}}\right]>E\left[U_{R_{x}}\right]$. This can easily be shown to occur whenever the expected value of the harms from nonresponse are larger than the cost of the companion diagnostic

$$
(1-R)[p-C]>P .
$$




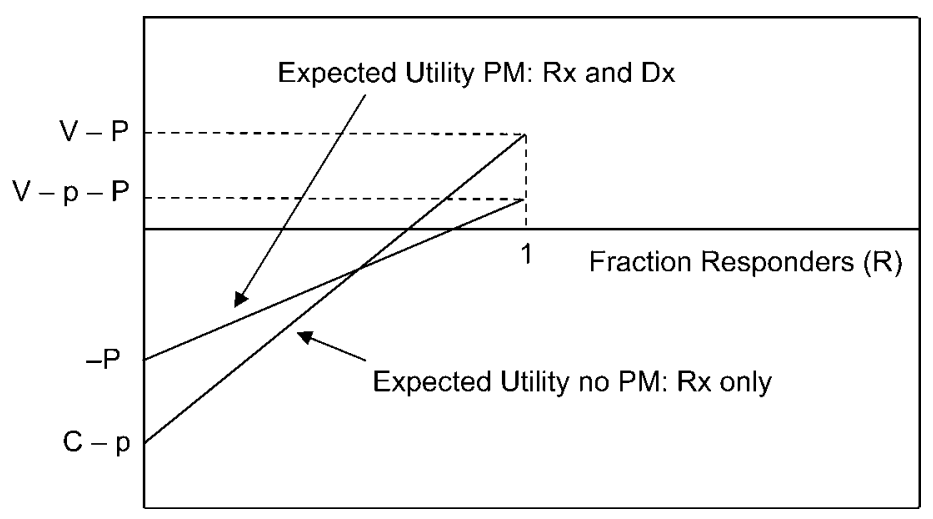

Fig. 1.3 Value of personalized medicine as a function of response rate

The harms from nonresponse involve paying for ineffective therapy as well as forgoing alternative care. Note that for the responders the value with PM is lower than without PM, as can be seen by the PM curve being below the non-PM curve at $R=1$; that is, the inequality above never holds if $R=1$. This is because responders just learn from the Dx what they would have learned less expensively through consumption.

Ex ante, all are better off from the value of the information generated by the companion diagnostic. The higher of the two lines is the expected utility under optimal use of PM. Note that at higher response rates, no personalized medicine is optimal, and at lower response rates it is not optimal. This reflects that the ex post winners from PM are those that never use the PM, and the ex post losers from PM are those that use the PM. This occurs because the responders would have gone on treatment anyway and just paid the cost of the diagnostic without changing their treatment consumption.

Now consider when the expected utility from treatment $E\left[U_{R_{x}}\right]$ is negative so that without a companion diagnostic, no treatment is undertaken. The treatment may not even be allowed if, say, regulatory bodies such as the Food and Drug Administration deem the side effects $(C)$ more harmful than the benefits of the treatment $(V)$ or the set of responders $(R)$ too small. In this case, the companion diagnostic may open up the market for the treatment if nonresponders or those with severe side effects can be tested out. This scenario would be likely either when regulation or reimbursement required the use of diagnostics before using the treatment. In the regulatory case, the diagnostic would be a "rescue diagnostic" if the treatment failed regulatory approval, and in the reimbursement case copays would be higher without the diagnostic.

The expected utility with the companion diagnostic is the same as before

$$
E\left[U_{D_{x}}\right]=R \cdot(V-p)-P .
$$


However, now that the diagnostic is used, $E\left[U_{D_{x}}\right]>E\left[U_{R_{x}}\right]$, whenever

$$
R \cdot(V-p)>P .
$$

This occurs whenever the expected consumer surplus of testing in is larger than the cost of the test. By contrast from before, the ex post winners are the ones using the personalized treatments as they gain the surplus. The ex post losers are the ones testing out of treatment - in the absence of the diagnostic they would not undergo treatment, but would not pay for the diagnostic.

\subsection{Pricing of the Combination Product of Personalized Medicine}

In this section we discuss aspects of the optimal pricing of PM and how it affects the appropriation by innovators of the value generated by PM, and thus innovation incentives.

The value generated by PM comes from the combination of two products: a companion diagnostic used together with the treatment of interest. On a fundamental level this combination of products must make the patient better off, as the patient can always not use the companion diagnostic and be as well off as before, and therefore is an improvement in the quality of care. It is often argued that PM is a threat to innovative returns because markets will be smaller when patients test out of them using companion diagnostics. However, the fact that the quality of care is improved can often mean that innovators should be able to price that quality higher and potentially make larger, not smaller, profits through personalized medicines.

The key is to understanding when PM enhances profits and innovation incentives; greater total profits of both Rx and Dx may be enabled, even when Rx profits alone may fall. More precisely, let the total profits of the two products be denoted

$$
\Pi(p, P)=\pi_{R_{x}}(p, P)+\pi_{D_{x}}(p, P) .
$$

Under joint ownership of the two products, the joint owner can always price them as separate owners would. However, separate owners of the two products do not take into account the negative external effects on the profits of the other product when setting their own price. Thus, the profits must always be highest under joint ownership internalizing the pricing impacts of one product on the profits of the other. Put simply, the monopoly profits of the joint owner must be higher than the total profits of two separate owners, creating merger incentives between $\mathrm{Rx}$ and Dx owners. This is a well-known result from optimal monopoly pricing under interdependent demand and has been pointed out in the context of stratified medicine by Trusheim, Berndt, and Douglas (2007).

The spillover in profits across the two products occurs whenever the two have interdependent demands. This occurs particularly whenever the $\mathrm{Rx}$ and Dx are substitutes or complements. Our arguments stressed that they were 


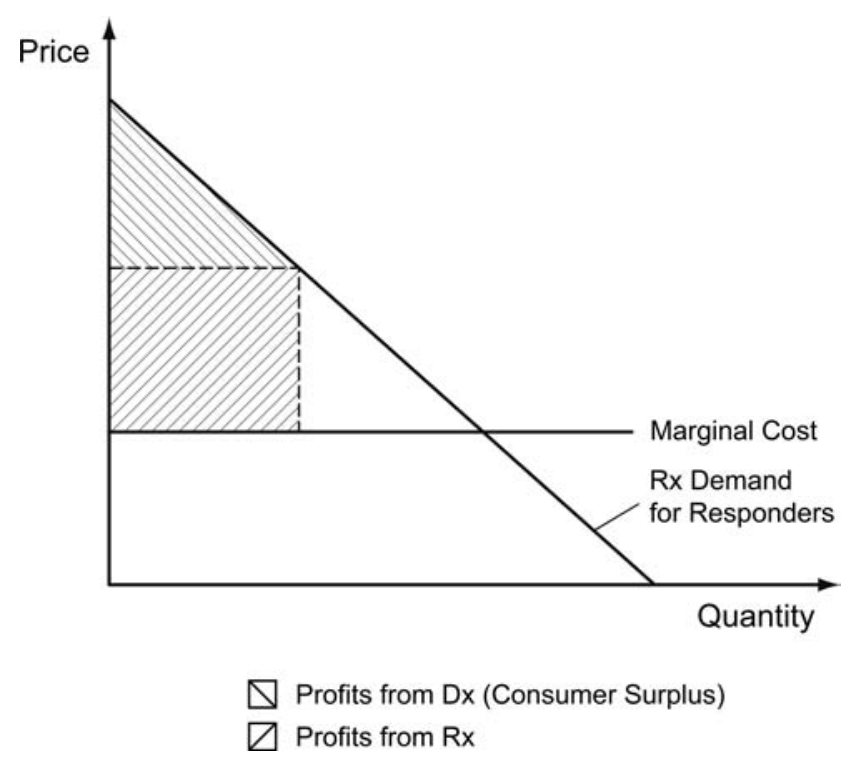

Fig. 1.4 Two-part pricing of personalized medicine

inherently substitutes in terms of providing two different methods of learning about treatment response. However, the two are likely to be complements due to either regulation or reimbursement policies. This is because most approval agencies or payers will likely only allow the drug to be consumed or allow lower copays after having tested into therapy through the companion diagnostic. This thus requires the two to be consumed together in that entry into the therapy can only come through first taking the diagnostic.

When the two products are regulatory complements in this way, in that the $\mathrm{Rx}$ can only be consumed when the Dx has, the pricing of the two resembles a two-part pricing problem similar to the classic Disneyland pricing problem discussed by Oi (1971). In this analogy, the Dx is the "gate" of the park and the Rx is the "ride" once inside the park. Figure 1.4 illustrates the optimal joint pricing of the products under joint ownership.

The joint owner can capture the entire (expected) consumer surplus by selling the drugs at marginal cost and pricing the Dx at the expected consumer surplus from using the drug. Just as for Disneyland, the gate price should be high and ride price low.

As is common for two-part pricing problems, this full extraction of the social surplus to the joint owner may not be feasible when consumers are heterogeneous. The implicit homogeneous assumption is important because under heterogeneity optimal pricing does not necessary imply that PM increases social surplus, though it must increase producers' surplus and thus incentives for $\mathrm{Rx}$ innovation. It is true that total profits from $\mathrm{Rx}$ and $\mathrm{Dx}$ 
are higher with the presence of a companion Dx, since the joint owner can always price the Dx out of the market, generating zero demand for it and yielding the Rx profits without the Dx feasible but not necessarily optimal.

However, the full appropriation of the value of PM to the innovator may not be feasible due to other constraints imposed by intellectual property issues and reimbursement policy in many countries. For example, in the United States companion tests are often sold at cost through laboratory test fee schedules, which prevents value-based pricing. In addition, intellectual property is often not fully protected for these products through various court decisions.

\subsection{Conclusion}

This chapter considers the value and optimal pricing of PM stemming from the value of information contained in companion diagnostics. We argue that PM provided new treatment-specific learning innovations that continue the quest to better match treatments to patients and that the information value PMs provide prevents inefficient over- and underconsumption by nonresponders and responders. Personalized medicine converts treatments from being experience goods to search goods, which helps explain their emergence in cancer care where learning through experience is the most costly as it can involve inefficient mortality, especially with the growing number of emerging alternative treatments. We argue that the incidence of the value of these new technologies is such that everyone gains ex ante, but that those who undertake personalized therapies could often be ex post losers from PM. We discuss the optimal two-part pricing of PMs when they are regulated complements mandating they be consumed together.

Personalized medicine is not only closely linked to learning-driven adherence, but also to pay for performance ( $\mathrm{P} 4 \mathrm{P})$ issues, which future research should better analyze. Reimbursing for a companion diagnostic is essentially a P4P scheme that aims to undertake treatment spending only on responders. It is, therefore, very closely linked to standard P4P schemes that use treatment experience as the mechanism by which treatment performance or quality is assessed. Standard P4P schemes essentially aim to compensate sellers only if the product performs well. This basically boils down to the classic practice of using initial discounts, warranties, or money-back guarantees for experience goods. There is a literature on pricing of experience goods that shows the conditions under which initial discounts are optimal in order to subsidize the learning of consumers (see, e.g., Bergemann and Välimäki 2006). For medical products, this amounts to sellers providing samples free of charge to providers and patients. A better understanding of how such pricing of experience goods compares to simply paying for the companion diagnostics of PM is needed. Both seek to avoid paying for nonperformance, but differ in that PM does not necessarily require the undertaking of treat- 
ment to determine such performance. In addition, if learning drives adherence, then patients do not adhere when a therapy does not perform, and thus patients themselves engage in pay for performance, which is distinguished from $\mathrm{P} 4 \mathrm{P}$ schemes of payers. Here P4P schemes by payers to manufacturers may have small effects as payers do not pay for ineffective care when patients do not adhere to it.

Further analysis of the relationships among adherence, PM, and P4P is clearly needed and will better inform the policy debate on reimbursement and regulations of PM. What has been missing from the economic analysis of PM is the relative comparative advantage compared to trial and error in learning about suitable patient and treatment matches. Recognizing this incremental value of learning from diagnostics versus experience seems central to determining their value and forming reimbursement policies driving the incentives to using PMs and bringing them to market.

\section{References}

Bergemann, Dirk, and Juuso Välimäki. 2006. "Dynamic Pricing of New Experience Goods." Journal of Political Economy 114 (4): 713-43.

Egan, M., and T. Philipson. 2015. "Health Care Adherence and Personalized Medicine.” NBER Working Paper no. 20330, Cambridge, MA.

Food and Drug Administration (FDA). 2017. "Table of Pharmacogenomic Biomarkers in Drug Labels." https://www.fda.gov/Drugs/ScienceResearch/ucm572698 .htm.

Oi, Walter Y. 1971. "A Disneyland Dilemma: Two-Part Tariffs for a Mickey Mouse Monopoly." Quarterly Journal of Economics 85 (1): 77-96.

Philipson, Tomas, and Jeffrey DeSimone. 1997. "Experiments and Subject Sampling." Biometrika 84 (3): 619-30.

Sood, Neeraj, Tomas J. Philipson, and Peter Huckfeldt. 2013. "Quantifying the Value of Personalized Medicines: Evidence from COX-2 Inhibitors." Forum for Health Economics \& Policy 16 (1): 101-22.

Trusheim, Mark R., Ernst R. Berndt, and Frank L. Douglas. 2007. "Stratified Medicine: Strategic and Economic Implications of Combining Drugs and Clinical Biomarkers." Nature Reviews Drug Discovery 6:287-93.

Tufts Center for the Study of Drug Development. 2015. "Personalized Medicine Gains Traction But Still Faces Multiple Challenges.” Impact Report 17 (3). https:// csdd.tufts.edu/impact-reports/.

Yeaw, Jason, Joshua S. Benner, John G. Walt, Sergey Sian, and Daniel B. Smith. 2009. "Comparing Adherence and Persistence across 6 Chronic Medication Classes." Journal of Managed Care Pharmacy 15 (9): 728-40. 